\title{
ANÁLISIS DE LA PROGENIE DE INDIVIDUOS PRODUCTORES Y NO PRODUCTORES DE GAMETOS MASCULINOS NO REDUCIDOS DE TURNERA SIDOIDES (PASSIFLORACEAE)
}

\author{
IVANA EVELIN KOVALSKY ${ }^{1,2}$ y VIVIANA G. SOLÍS NEFFA ${ }^{1,2}$
}

\begin{abstract}
Resumen: La unión de gametos $2 n$ ha tenido un importante papel en el origen de los poliploides de Turnera sidoides. Sin embargo, como en este complejo la tasa de formación de neopoliploides es baja, el establecimiento de los mismos constituiría una etapa crítica. Considerando que la dinámica evolutiva de los poliploides depende en gran medida de la frecuencia de producción de gametos $2 n$, dicha etapa podría ser superada si la capacidad de los diploides de producir estos gametos es transmitida a la descendencia. En este marco, se analizó la progenie de cruzamientos experimentales entre productores y no productores de gametos masculinos $2 n$ de $T$. sidoides. Los resultados mostraron que la capacidad de producir gametos $2 n$ estaría regulada por un gen con dos alelos, uno de ellos completamente dominante sobre el otro, siendo el carácter recesivo. La variación en la frecuencia de los gametos $2 n$ y $4 n$ sería atribuida a diferencias en la expresión génica. La posibilidad de transmitir la capacidad de producir gametos masculinos $2 n$ a la progenie, contribuiría a aumentar tanto las tasas de formación como la probabilidad de que ocurran cruzamientos entre individuos productores de estos gametos, favoreciendo el establecimiento de neopoliploides en las poblaciones diploides del T. sidoides.
\end{abstract}

Palabras clave: Turnera sidoides, gametos masculinos no reducidos, poliploidía, cruzamientos experimentales.

\begin{abstract}
Summary: Analysis of the progeny of unreduced male gametes producers and non-producers of Turnerasidoides (Passifloraceae). The union of $2 n$ gametes has played an important role in the origin of polyploids of Turnera sidoides. Notwithstanding, owing to the lower rate of neopolyploid formation in this complex, their establishment constitute a critical step. Considering that the evolutionary dynamics of polyploids depends in great part on the frequency of $2 n$ gametes production, such step would be overcome if the capacity of $2 n$ gametes production is transmitted to the progeny. In this context, the progeny of experimental crosses between producers and non producers of $2 n$ gametes of $T$. sidoides was analyzed. The results showed that the capacity of $2 n$ gametes production would be regulated by one locus with two alleles, one of them completely dominant, being the trait recessive. The variation in the frequency of $2 n$ and $4 n$ gametes detected in the progeny would be attributed to differences in gene expression. The possibility of transmitting to the progeny the capacity of $2 n$ male gametes production, would contribute to enhance the rate of polyploid formation as well as the likelihood of crossings between producers of the $2 n$ gametes, favoring the establishment of neoplyploids in diploid populations of $T$. sidoides.
\end{abstract}

Key words: Turnera sidoides, unreduced male gametes, polyploidy, experimental crosses.

\section{INTRODUCCIÓN}

La poliploidización sexual como resultado de la unión de gametos no reducidos, es considerada el principal mecanismo de origen de nuevos poliploides

${ }^{1}$ Laboratorio de Citogenética y Evolución Vegetal. Instituto de Botánica del Nordeste (UNNE-CONICET). C.C. 209, 3400, Corrientes, Argentina.

${ }^{2}$ Facultad de Ciencias Exactas y Naturales y Agrimensura (UNNE). E-mail: evelinkov@yahoo.com.ar en las poblaciones naturales de plantas (Harlan \& de Wet, 1975; de Wet, 1980; Camadro, 1986, 1994; Bretagnolle \& Thompson, 1995;Ramsey \& Schemske, 1998). Debido a fallas en la meiosis, en dichos gametos el número cromosómico no se reduce (2n) o incluso se duplica (4n) (Mercieret al., 2001, 2003; Agasheet al., 2002; Boatenget al., 2008; d' Erfurt et al., 2008, 2009, 2010; Brownfield \& Köler, 2011; Schatlowski \& Köler, 2012). Por lo tanto, al intervenir en la fecundación, dan lugar a la formación de individuos poliploides en uno (poliploidización 
sexual bilateral) o dos pasos (poliploidización sexual unilateral) (Ramsey \& Schemske, 1998).

Debido a la importante función de los gametos no reducidos en el origen de los poliploides así como en el mejoramiento vegetal, numerosos trabajos se han centrado en la detección de dichos gametos (Mendiburu \& Peloquín, 1977; Parrot \& Smith, 1985; Orjeda et al., 1990; Pan et al., 2004; Dewitte et al. 2009; De Haan et al., 1992; De Storme et al., 2011, 2013; Veerle et al., 2002).Asimismo, debido a que la tasa de formación de poliploides depende de la frecuencia de producción de gametos no reducidos (Bretagnolle \& Thompson, 1995; Ramsey \&Shemske, 1998), el análisis de la producción de este tipo de gametos también resulta relevante para la compresión de los factores que influyen en el establecimiento de los poliploides y en la dinámica de las poblaciones mixtas diploide-tetraploide (Levin, 1983; Fowler \& Levin, 1984). En este sentido, se ha sugerido que tanto los factores genéticos (Hermsen, 1984; Veilleux, 1985; Bretagnolle \& Thompson, 1995; Qu \& Vorsa, 1999; d'Erfurth et al., 2008) como los ambientales (Maizonnier, 1976; Veilleux \& Lauer, 1981; Kaul \& Murthy, 1985; Watanabe \& Peloquín, 1989) pueden influir en la producción de los gametos no reducidos y, en consecuencia, en la probabilidad de origen de neopoliploides por poliploidización sexual. Por otra parte, debido a que se trata de un carácter heredable (Mc Coy, 1982; Parrot \& Smith, 1985; Tavoletti et al., 1991; Ortiz \& Peloquín, 1992; Ortiz, 1996), la posibilidad de que los individuos diploides productores de gametos no reducidos transmitan la capacidad de producir dichos gametos a la progenie, también podría aumentar la probabilidad de origen y de establecimiento de los neopoliploides en las poblaciones naturales.

Turnera L. (Passifloraceae) constituye un modelo adecuado para investigar la dinámica evolutiva de los poliploides, ya que la poliploidía constituye un fenómeno muy frecuente en este género. Aproximadamente el $59 \%$ de las poblaciones analizadas hasta el momento son de origen poliploide, hallándose desde triploides hasta decaploides (Fernández, 1987; Solís Neffa \& Fernández, 2000, 2001; Solís Neffa et al., 2004; Elías et al., 2011; Kovalsky \& Solís Neffa, 2012). Los estudios citogenéticos en diversas especies mostraron que existen tanto alopoliploides como autopoliploides (Fernández, 1987; Fernández \& Arbo, 1990, 2000a y b; Solís Neffa, 2000).
La poliploidización sexual ha tenido un importante papel en la dinámica evolutiva de los poliploides de Turnera, habiéndose detectado la producción de gametos no reducidos en poblaciones naturales diploides de diferentes especies (Panseri et al., 2008; Lazaroff et al., 2010; Kovalsky \& Solís Neffa, 2012) y en híbridos interespecíficos (Fernandez \& Arbo, 1990; Fernández et al., 2010). En particular, en el complejo autopoliploide $T$. sidoides $\mathrm{L} .(\mathrm{x}=7)$ se ha investigado exhaustivamente la producción de gametos no reducidos en poblaciones naturales (Kovalsky, 2012; Kovalsky \& Solís Neffa, 2012). Dichos estudios demostraron que la producción de gametos no reducidos no es infrecuente en este complejo y en el género. Asimismo, teniendo en cuenta que algunas plantas poseen la capacidad de producir este tipo de gametos mientras que otras no y, que las plantas que fueron identificadas como productoras en el campo mantuvieron la capacidad de producir gametos no reducidos luego de dos años de cultivo en condiciones homogéneas de invernáculo, se ha sugerido que la producción de gametos masculinos no reducidos estaría regulada genéticamente. Por otra parte, dado que la tasa estimada de formación de neopoliploides en $T$. sidoideses baja, el establecimiento de los mismos constituiría una etapa crítica (Kovalsky \& Solís Neffa, 2012). En estas circunstancias, si la capacidad de los diploides de producir gametos no reducidos es transmitida a la descendencia, el aumento de los cruzamientos entre individuos relacionados productores de gametos no reducidos podría favorecer el establecimiento y la persistencia de los neotetraploides en las poblaciones diploides de $T$. sidoides.

Sobre la base de estos antecedentes y a fin de contribuir a la comprensión de los mecanismos de origen y establecimiento de neopoliploides, en este trabajo se analiza la producción de gametos masculinos no reducidos en la progenie de cruzamientos experimentales realizados entre individuos productores y no productores de gametos masculinos no reducidos de T. sidoides.

\section{Materiales y Métodos}

Se llevaron a cabo cruzamientos experimentales entre individuos productores y no productores de gametos masculinos no reducidos provenientes de 


\section{E. Kovalsky y V. G. Solís Neffa - Análisis de progenie de Turnera sidoides}

la población $\mathrm{S}_{215}$ de Turnera sidoides subsp. carnea (Cambess.) Arbo, localizada en la ruta 123 y el río Miriñay $\left(29^{\circ} 33^{\prime} 44,0^{\prime \prime} \mathrm{S}, 57^{\circ} 30^{\prime} 40,0^{\prime}\right.$ ' $\mathrm{O}, 66$ m s.n.m.), en el departamento de Mercedes de la provincia de Corrientes (Argentina). El ejemplar testigo (Solís Neffa \& Seijo 960) se encuentra depositado en el Herbario del Instituto de Botánica del Nordeste (CTES). Algunas plantas fueron transportadas a la ciudad de Corrientes donde se cultivaron bajo condiciones controladas en el invernáculo del mismo instituto. Se seleccionó esta población, debido a que en estudios previos se detectó una alta producción de gametos masculinos no reducidos (Panseri et al., 2008; Kovalsky \& Solís Neffa, 2012) y se analizaron los mecanismos citológicos que conducen a la formación de estos gametos (Kovalsky et al., 2014).

\section{Cruzamientos controlados}

Teniendo en cuenta que $T$. sidoides es distila (Arbo, 1985) y que los cruzamientos legítimos, es decir los que se producen entre flores longistilas (L) y brevistilas (B) son los únicos viables (Solís Neffa, 2000), se seleccionaron plantas L y B productoras de gametos masculinos reducidos $(n)$ y altas productoras de gametos masculinos no reducidos de la población $\mathrm{S}_{215}$ para llevar a cabo los cruzamientos recíprocos. Para la obtención de la $\mathrm{F}_{2}$, se llevaron a cabo cruzamientos entre plantas L y B productoras de gametos masculinos reducidos y no reducidos de la $\mathrm{F}_{1}$.

Aunque T. sidoides es autoincompatible (Solís Neffa, 2000), las flores de las plantas utilizadas como progenitores femeninos fueron castradas previamente a la polinización con el polen de la planta seleccionada como progenitor masculino. Las flores polinizadas se marcaron con una etiqueta indicando el número de cruzamiento y los frutos en desarrollo se encerraron en una bolsa de tul de malla fina para asegurar la cosecha de las semillas. Las semillas obtenidas se sembraron en macetas individuales y los individuos resultantes fueron transplantados luego de haberse desarrollado el primer par de hojas.

Tanto para la obtención de la $\mathrm{F}_{1}$ como de la $\mathrm{F}_{2}$, el número de flores polinizadas fue diferente para cada tipo de combinación parental empleada en los cruzamientos, dependiendo de la disponibilidad de plantas y de la floración simultánea de las plantas L y B.

\section{Nivel de ploidía de la progenie}

El nivel de ploidía de la progenie fue estimado por citometría de flujo a partir de hojas de los individuos adultos siguiendo las recomendaciones para la preparación de las muestras de Partec UV kit CyStain Precise P (05-5002). Se colocaron $0,5 \mathrm{~cm}^{2}$ de hojas frescas en una cápsula de Petri con cantidades comparables de hojas de la planta empleada como patrón (el individuo $\mathrm{S}_{215}-48$ ) cuyo nivel de ploidía fuera estimado previamente por recuentos en meiosis (Kovalsky \& Solís Neffa, 2012). Tras añadir $0,5 \mathrm{ml}$ de buffer de extracción las muestras fueron trituradas con una hoja de afeitar. Después de 2 min de incubación, el homogenado fue filtrado a través de una de malla de nylon de $50 \mu \mathrm{m}$ de apertura y colocadas en un tubo donde se añadieron $1,5 \mathrm{ml}$ de buffer de tinción DAPI (4',6-diamidino-2-fenilindol). La mezcla fue incubada durante $2 \mathrm{~min}$ a temperatura ambiente y luego analizada. Se midió la intensidad de fluorescencia de los núcleos teñidos con DAPI mediante un citómetro de flujo Partec PA II (PartecGmbH, Münster, Alemania) con el detector funcionando a $355 \mathrm{~nm}$. Se midieron alrededor de 5000 núcleos por muestra. El nivel de ploidía de cada muestra se estimó comparando el pico de ADN de dicha muestra con el pico del patrón. El análisis de los datos se realizó mediante el software PA II PartecFlomax.

Producción de gametos masculinos no reducidos en la progenie diploide

En la progenie diploide, los productores de gametos masculinos no reducidos fueron detectados mediante el análisis del tamaño de los granos de polen. Para tal fin, los mismos fueron extraídos de las anteras y coloreados con carmín glicerina (1:1). Debido que en T. sidoides el tamaño del polen varía entre las flores L y B (Solís Neffa, 2000; Panseri, 2011), el análisis fue realizado de forma independiente en ambos tipos florales. A partir de los datos obtenidos, se estimaron las frecuencias de individuos productores y no productores de gametos masculinos $2 n$.

En los individuos productores, se estimó la frecuencia de producción de gametos no reducidos. Para ello, en primer término, se midieron 150 granos por antera en cada una de las cinco anteras de tres flores por individuo. 
Para cada grano se estimó el tamaño empleando el índice Em; i.e. la distancia entre el ángulo apertural y el punto medio del lado opuesto (Van Campo, 1957). A partir de estas mediciones, se calculó para cada planta: la media, la desviación estándar y la distribución del tamaño de polen. Posteriormente, todos los granos de polen de cada antera fueron clasificados según su tamaño en tres clases: normal, gigante o $2 n$ (tamaño 1,25 veces superior al valor promedio del individuo) y jumbo o $4 n$ (tamaño 1,50 veces superior al valor promedio del individuo). Luego, para cada individuo analizado, se estimó la frecuencia de las tres clases de polen dividiendo el número de granos normal, gigante y jumbo por el número total de granos de polen. El valor promedio de la producción de gametos no reducidos en plantas $\mathrm{L}$ y B de la progenie fue comparado con el de sus progenitores mediante un análisis de la varianza (ANOVA) con un nivel de significación del $5 \%$ $(\alpha=0,05)$.

Finalmente, se analizó el ajuste de las frecuencias de individuos productores y no productores observadas en la progenie, a las esperadas bajo dos modelos alternativos de herencia mediante un test de $\mathrm{X}^{2}$ de bondad de ajuste. Las hipótesis a comprobar fueron que la capacidad de producir gametos masculinos no reducidos depende de un locus con dos alelos, uno de ellos completamente dominante sobre el otro, estando el carácter determinado por un alelo recesivo (hipótesis 1) o por un alelo dominante (hipótesis 2).

Los análisis estadísticos fueron realizados empleando el programa Infostat versión 2014 (Di Rienzo et al., 2014).

\section{Resultados}

\section{Cruzamientos controlados}

Los resultados de los 133 cruzamientos realizados se muestran en la Tabla 1 y en la Fig. 1.

\section{Nivel de ploidía de la progenie}

Tanto en la $\mathrm{F}_{1}$ como en la $\mathrm{F}_{2}$ se hallaron individuos diploides, pero en la $\mathrm{F}_{2}$ en los cruzamientos en los cuales ambos progenitores eran productores de gametos masculinos no reducidos, además de diploides también se detectaron triploides (Tabla 1).

\section{Producción de gametos masculinos no reducidos}

El tamaño promedio del polen fue mayor en la $\mathrm{F}_{2}$, comparado con la $\mathrm{F}_{0}$ y la $\mathrm{F}_{1}$. En los parentales, el tamaño promedio del polen fue de $38,60 \mu \mathrm{m} \pm$ $4,90 \mu \mathrm{m}(29,40 \mu \mathrm{m}-50,96 \mu \mathrm{m})$ en las flores $\mathrm{L} y$ $45,88 \mu \mathrm{m} \pm 4,25 \mu \mathrm{m}(31,36 \mu \mathrm{m}-68,60 \mu \mathrm{m})$, en las B. En la $F_{1}$ el tamaño promedio del polen fue de $37,66 \mu \mathrm{m} \pm 4,33 \mu \mathrm{m}(29,40 \mu \mathrm{m}$ y $58,80 \mu \mathrm{m})$ у $47,76 \mu \mathrm{m} \pm 3,85 \mu \mathrm{m}(33,32 \mu \mathrm{m}-72,52 \mu \mathrm{m})$ en las flores L y B, respectivamente. Finalmente, en la $F_{2}$ el tamaño promedio del polen de las flores L fue de $38,72 \mu \mathrm{m} \pm 5,38 \mu \mathrm{m}(29,40 \mu \mathrm{m}-60,76$ $\mu \mathrm{m})$ y en las B fue de $46,92 \mu \mathrm{m} \pm 4,26 \mu \mathrm{m}(31,36$ $\mu \mathrm{m}-72,52 \mu \mathrm{m})$.

El porcentaje de productores de gametos no reducidos y la frecuencia de producción de los gametos $n, 2 n$ y $4 n$ de los parentales y las generaciones $\mathrm{F}_{1}$ y $\mathrm{F}_{2}$ se muestran en la Tabla 1. En ambas generaciones, se hallaron plantas (L y B) productoras de gametos masculinos no reducidos. Se detectaron diferencias significativas en la frecuencia de producción de gametos no reducidos entre los individuos parentales y la progenie, en ambos tipos florales ( $\mathrm{L}: \mathrm{F}=3,89 \mathrm{p}=0,04$; $\mathrm{B}$ : $\mathrm{F}=145,5 \mathrm{p}<0,0001)$. En general, el porcentaje individuos productores y de frecuencia de producción de gametos masculinos no reducidos fue mayor en la $\mathrm{F}_{2}$. Asimismo, tanto en la $\mathrm{F}_{1}$ como en la $\mathrm{F}_{2}$, los mayores porcentajes de individuos productores y de gametos $2 n$ y $4 n$ se obtuvieron en los cruzamientos en los cuales el progenitor masculino o ambos, eran productores de gametos masculinos no reducidos. Tanto en la $F_{1}$ como en la $\mathrm{F}_{2}$, en la mayoría de los individuos productores se detectaron tanto gametos $2 n$ como $4 n$, excepto en los individuos productores de la progenie de los cruzamientos en los cuales el productor de gametos masculinos no reducidos fue el progenitor femenino, los que sólo produjeron gametos $n$ y $2 n$, pero no $4 n$. Los mayores porcentajes de individuos productores así como de gametos $2 n$ y $4 n$ producidos por individuo fueron observados en los cruzamientos de la $\mathrm{F}_{2}$ en los que ambos progenitores eran productores de gametos masculinos no reducidos.

El análisis del ajuste de los valores observados a las proporciones esperadas bajo los dos modelos alternativos de herencia, reveló que para la hipótesis 1 (carácter recesivo) $X^{2}=0,0066$; mientras que para la hipótesis 2 (carácter dominante) $\mathrm{X}^{2}=69,36$. 
Tabla 1. Variación de la frecuencia de producción de gametos masculinos no reducidos en F0, F1 y F2 de cruzamientos experimentales en Turnera sidoides subsp. carnea.

\begin{tabular}{|c|c|c|c|c|c|c|c|c|}
\hline \multirow[b]{2}{*}{ Cruzamiento } & & \multirow[t]{2}{*}{$F_{0}$} & \multicolumn{3}{|c|}{$\mathrm{F}_{1}$} & \multicolumn{3}{|c|}{$\mathbf{F}_{2}$} \\
\hline & & & $\mathbf{n} \times \mathbf{2 n}$ & $2 n \times n$ & $2 n \times 2 n$ & $\mathbf{n} \times \mathbf{2 n}$ & $2 n \times n$ & $2 n \times n$ \\
\hline $\begin{array}{l}\text { Número de } \\
\text { cruzamientos }\end{array}$ & & & 20 & 18 & 21 & 16 & 18 & 40 \\
\hline \multirow{2}{*}{$\begin{array}{c}\text { Nivel de ploidía de la } \\
\text { progenie (\%) }\end{array}$} & $2 \mathrm{x}$ & 100 & 100 & 100 & 100 & 100 & 100 & 93,06 \\
\hline & $3 x$ & 0 & 0 & 0 & 0 & 0 & 0 & 6,94 \\
\hline $\begin{array}{l}\text { Productores de } \\
\text { gametos } 2 n(\%)\end{array}$ & & & 40 & 25 & 66 & 50 & 50 & 100 \\
\hline \multirow{3}{*}{$\begin{array}{l}\text { Tipo de gametos } \\
\text { producidos (\%) }\end{array}$} & $n$ & 98,64 & 94,59 & 99,11 & 94,33 & 88,8 & 99,35 & 90,96 \\
\hline & $2 n$ & 1,26 & 5,3 & 0,89 & 3,99 & 7,9 & 0,65 & 2,88 \\
\hline & $4 n$ & 0,1 & 0,11 & 0 & 1,68 & 3,3 & 0 & 6,16 \\
\hline
\end{tabular}
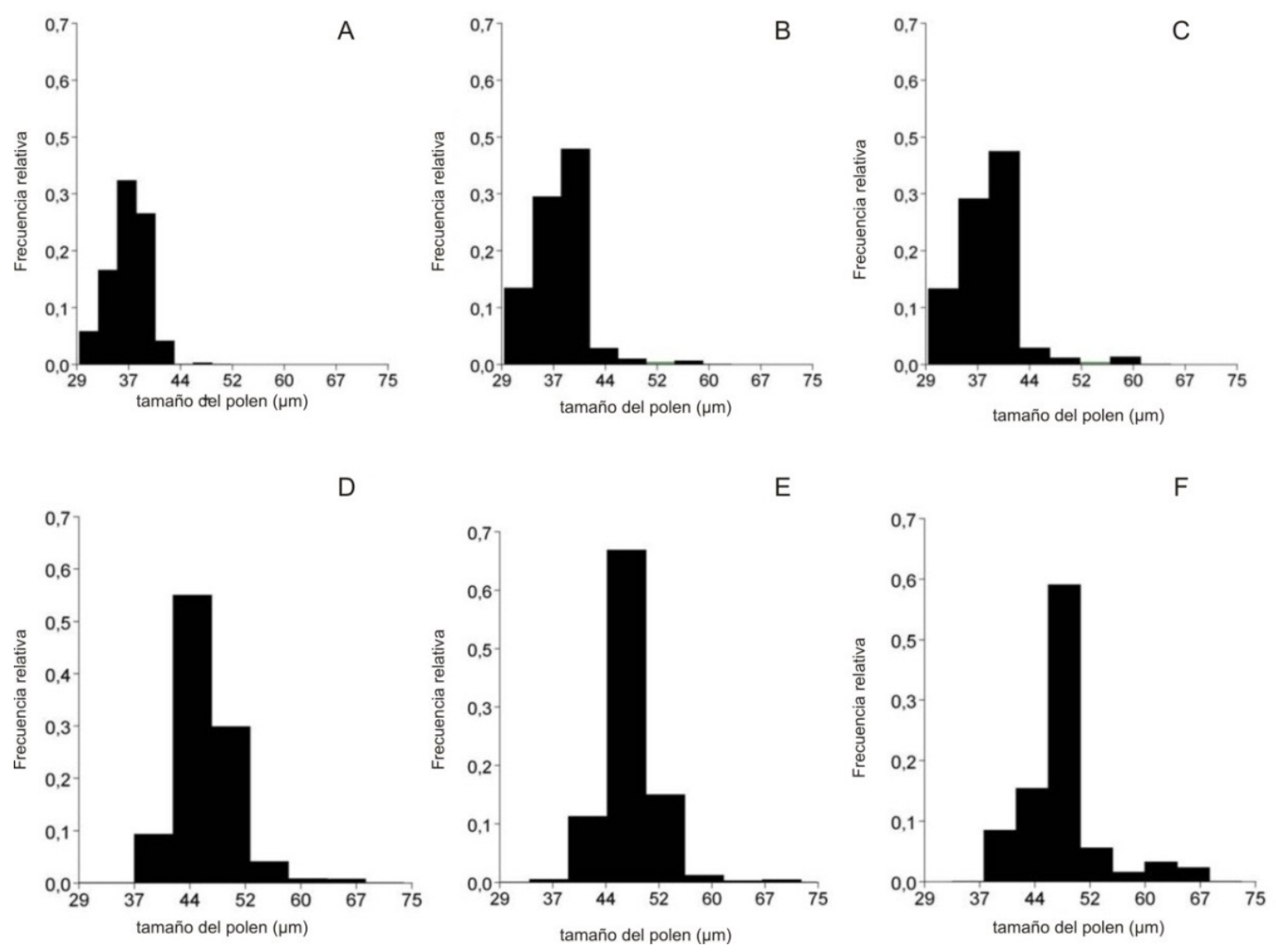

Fig. 1. Variación del tamaño del polen en los progenitores y en la progenie de plantas productoras de gametos no reducidos de Turnera sidoides. A, B y C, Flores longistilas. A, F0. B, F1.C, F2. D, E y F, Flores brevistilas. D, F0. E, F1. F, F2. 


\section{Discusión}

Los resultados obtenidos de los cruzamientos experimentales entre individuos productores y no productores de gametos masculinos no reducidos demuestran que, en T. sidoides, la capacidad de producir gametos no reducidos está regulada genéticamente, aunque diferencias en la expresión génica determinarían la variación en la frecuencia de producción de gametos masculinos no reducidos detectada en la progenie.

Diversas investigaciones demostraron que la formación de los gametos no reducidos en plantas está regulada por genes mutantes, que pueden actuar durante la cariocinesis de la meiosis (Ramanna, 1983, 1992; Hermsen, 1984; Parrot \& Smith, 1985; Kaul \& Murthy, 1985; Veilleux, 1985; Katsiotis \& Forsberg, 1995; Mercier et al., 2001, 2003; Agashe et al., 2002; Boateng et al., 2008; d' Erfurth et al., 2008; d'Erfurth, 2009; Erilova et al., 2009; Schatlowski \& Köler, 2012), la citocinesis (Mc Coy, 1982; Tavoletti et al., 2000) o en las distintas etapas del ciclo celular (Brownfield \& Köler, 2011; Schatlowski \& Köler, 2012). Dichos mutantes son generalmente recesivos, aunque en Musa L. (Ortiz, 1996) la capacidad de producir gametos no reducidos estaría determinada por un alelo mutante dominante. La mayoría de los mutantes meióticos actúan tanto en la microsporogénesis como en la megasporogénesis (Jongedijk \& Ramanna, 1988, 1989; Jongedijk et al., 1990;Erilova et al., 2009), aunque algunos sólo operan en la microsporogénesis (d'Erfurth et al., 2008). Asimismo, se ha sugerido que cada uno de los mecanismos citológicos por los cuales se forman los gametos masculinos no reducidos, estaría controlado por un gen con un alelo mutante recesivo. Los individuos que son homocigotos recesivos en más de uno de esos loci, producirían gametos no reducidos por más de un mecanismo, aunque en cada célula madre del polen solamente se expresaría uno de ellos (Mok \& Peloquín, 1975; Katsiotis \& Forsberg, 1995; Schatlowski \& Köler, 2012).

En T. sidoides, las proporciones de productores y no productores observados en las generaciones $F_{1}$ y $\mathrm{F}_{2}$ sugieren que la capacidad de producir gametos masculinos no reducidos estaría regulada por un gen con dos alelos, uno de ellos completamente dominante sobre el otro, estando el carácter determinado por el alelo recesivo. Sin embargo, dado que algunos individuos de T. sidoides sólo producen gametos masculinos $n$ y $2 n$, mientras que otros producen gametos $n, 2 n$ y $4 n$, y que se ha demostrado que diferentes mecanismos citológicos estarían implicados en la formación de dichos gametos (Kovalsky et al., 2014), es posible que cada uno de estos mecanismos esté regulado por un gen y que la producción de gametos $2 n$ y/o $4 n$ se deba a la presencia en homocigosis de los alelos recesivos en uno o más de dichos genes.

Asimismo, la variación en la frecuencia de los gametos $2 n$ y $4 n$ producidos por los individuos diploides de $T$. sidoides puede ser atribuida a diferentes factores. Se ha sugerido que los mutantes meióticos que determinan la producción de gametos no reducidos pueden presentar distintos grados de penetrancia y expresividad (Maizonnier, 1976; Veilleux \& Lauer, 1981; Veilleux et al., 1982; Hermsem, 1984; Veilleux, 1985; Kaul \& Murthy, 1985; Watanabe \& Peloquín, 1989; Bretagnolle \& Thompson, 1995). Por ejemplo, el mutante meiótico ps de las especies tuberosas diploides del género Solanum L., tiene penetrancia incompleta (Mok \& Peloquín, 1975) y su expresión puede ser afectada por la acción génica complementaria de, al menos, dos alelos modificadores dominantes con efectos aditivos (Ortiz \& Peloquín, 1992). En Trifolium pratense L. y en Petunia Juss. se comprobó que un gen sería el responsable de la formación de polen $2 n$ y que entre cuatro a seis genes modificadores controlarían su frecuencia (Maizonnier, 1976; Parrot \& Smith, 1985; Jongedijk et al.,1990); mientras que en Medicago sativa L. se sugirió que los genes responsables de la formación de polen jumbo tendrían un efecto pleiotrópico (Veronessi et al., 1990). Además, en otras especies se ha demostrado que la frecuencia de producción de gametos no reducidos sería en parte dependiente del genotipo y del ambiente (Maizonnier, 1976; Veilleux \& Lauer, 1981; Kaul \& Murthy, 1985; Veilleux, 1985; Watanabe \& Peloquín, 1989). Por lo tanto, la variación en la frecuencia de producción de gametos no reducidos masculinos entre individuos, entre flores del mismo individuo, e incluso entre las anteras de una misma flor observada en T. sidoides podría ser el resultado de la acción de un gen con penetrancia incompleta y expresividad variable. Sin embargo, dado que se ha sugerido que las altas temperaturas ocurridas en algunos meses durante el período de floración de $T$. sidoides afectarían la 


\section{E. Kovalsky y V. G. Solís Neffa - Análisis de progenie de Turnera sidoides}

microsporogénesis, aumentando la frecuencia de producción de gametos masculinos no reducidos (Kovalsky \& Solís Neffa, 2012), la expresión de los genes responsables de la formación de dichos gametos sería parcialmente dependiente del ambiente.

Por otra parte, considerando que la dinámica evolutiva de los poliploides depende en gran medida de la frecuencia de producción de gametos no reducidos (Fowler \& Levin, 1984), el hecho de que en $T$. sidoides esta capacidad sea heredable aumentaría la probabilidad de origen y establecimiento de neopoliploides dentro de las poblaciones diploides del complejo.

En las Angiospermas, las poblaciones poliploides se habrían originado a partir de individuos poliploides aislados originados por poliploidización sexual en las poblaciones diploides (Harlan \& De Wet, 1975). Una vez surgido un neopoliploide, la producción de las generaciones siguientes de poliploides, la frecuencia y composición de citotipos depende de factores tales como el sistema reproductivo y el grado de aislamiento reproductivo entre los citotipos (Ramsey \& Shemske, 1998). En las especies autógamas, los neotetraploides pueden producir progenie tetraploide por autofecundación; mientras que en las especies alógamas, la segunda generación de tetraploides se puede producir por cruzamientos entre tetraploides originados en eventos independientes. Alternativamente, si el aislamiento reproductivo entre los citotipos no es muy fuerte, las retrocruzas con los progenitores diploides pueden producir progenie triploide la que, en un segundo paso, puede contribuir a la formación de tetraploides por cruzamientos tanto con los diploides como con los tetraploides. Las nuevas generaciones de poliploides también se pueden formar por retrocruzas con un progenitor diploide productor de gametos no reducidos (Thompson \& Lumaret, 1992). Como resultado de estos cruzamientos, se puede mantener una frecuencia baja de poliploides en las poblaciones diploides. Sin embargo, la tasa de formación de neopoliploides puede aumentar si los diploides producen una frecuencia relativamente alta de gametos no reducidos (superior al $17 \%$ si la fertilidad y viabilidad de los citotipos es la misma) $o$, si ocurre un incremento considerable en la producción de gametos no reducidos inducido por mutación, cambios ambientales y/o parásitos
(Felber, 1991). La magnitud de estos procesos puede acentuarse en poblaciones pequeñas como resultado del azar (Fowler \& Levin, 1984).

En T. sidoides, el hallazgo de individuos triploides en algunas poblaciones diploides actuales, aunque en frecuencias relativamente bajas, demostró que la poliploidización es un proceso activo en este complejo (Elías et al., 2011; Kovalsky \& Solís Neffa, 2012). Dado que los triploides de $T$. sidoides no son completamente estériles y producen gametos viables $n$ y $2 n$, y teniendo en cuenta que esta especie es alógama, las nuevas generaciones de poliploides (triploides y tetraploides) podrían originarse por cruzamientos entre triploides o a partir de retrocruzas con los progenitores diploides productores de gametos no reducidos. No obstante, teniendo en cuenta que la capacidad de producir gametos no reducidos se transmite a la progenie, la frecuencia de este tipo de gametos $\mathrm{y}$, en consecuencia, la probabilidad de origen de neotetraploides en un solo paso, podrían aumentar luego de sucesivas generaciones. La tasa de formación espontánea de neopoliploides podría aumentar aún más ante ciertas condiciones ambientales durante el período de floración de $T$. sidoides, en los que se ha demostrado que la producción de gametos no reducidos puede aumentar hasta un $22 \%$ (Kovalsky \& Solís Neffa, 2012).

Asimismo, el aumento en la tasa de formación de neopoliploides podría favorecer el establecimiento y la persistencia de los mismos en las poblaciones diploides de Turnera sidoides. En este sentido, las condiciones que facilitan el establecimiento y la coexistencia de los neopoliploides en las poblaciones junto a sus progenitores diploides fueron objeto de numerosos estudios teóricos (Levin, 1975; Fowler \& Levin, 1984; Van Dijk \& Bijlsma, 1994; Rodríguez, 1996) y de simulación (Husband \& Schemske, 2000; Husband \& Sabara, 2003). De acuerdo con el modelo de exclusión del citotipo minoritario (Levin, 1975), cuando diploides y tetraploides están presentes en una misma población, solamente los cruzamientos entre plantas de un mismo nivel de ploidía contribuyen al mantenimiento de cada citotipo, siendo la exclusión del citotipo minoritario un proceso dependiente de la frecuencia de los apareamientos exitosos. Como el citotipo minoritario, inicialmente el tetraploide, tiene un menor potencial para cruzarse, produce 
un número menor de semillas por planta que el mayoritario (el diploide), aunque ambos tengan el mismo potencial reproductivo. Si ambos citotipos poseen requerimientos ecológicos similares, la composición de plantas adultas en la población será la correspondiente al banco de semillas. Como el citotipo minoritario está en desventaja reproductiva, la proporción de individuos en la población adulta será mayor que la existente en el banco de semillas a partir de la cual se desarrollará la próxima generación. En consecuencia, el citotipo minoritario declinará en las sucesivas generaciones hasta ser eliminado de la población. En este contexto, la producción de gametos $2 n$ por los diploides constituye un factor esencial en la dinámica de las poblaciones mixtas dado que posibilita la formación continua de nuevos tetraploides, aumentando sus posibilidades de establecimiento (Levin, 1983; Fowler \& Levin, 1984; Singh et al., 1990; van Dijk et al., 1992; Bretagnolle \& Thompson, 1995; Ramsey \& Shemske, 1998).

En las poblaciones mixtas de T. sidoides, como los diploides y neopoliploides no difieren en sus requerimientos ecológicos y dado que los triploides poseen baja viabilidad, se ha sugerido que casi todos los cruzamientos exitosos ocurrirían entre individuos con el mismo nivel de ploidía (Elías, 2010; Elías et al., 2011). En este contexto, el hecho que en $T$. sidoides, la capacidad de producir gametos masculinos no reducidos haya sido mayor en la progenie de los individuos productores de este tipo de gametos, sumado a que esta especie crece frecuentemente en poblaciones pequeñas y se dispersa por gravedad, la progenie de las plantas productoras de gametos no reducidos tenderían a concentrarse alrededor de la planta madre y, por lo tanto, aumentarían la probabilidad de la ocurrencia de cruzamientos entre plantas relacionadas productoras de gametos no reducidos. La formación continua de neotetraploides como resultado de las sucesivas retrocruzas entre los productores de gametos no reducidos y su progenie, favorecerían el establecimiento y la persistencia de dichos poliploides en las poblaciones diploides de $T$. sidoides. La capacidad de multiplicación vegetativa por rizomas también beneficiaría la fijación de los neopoliploides de este complejo.

El análisis en curso de la distribución espacial de los citotipos y de los productores de gametos no reducidos en las poblaciones mixtas de Turnera sidoides contribuirán a una mejor comprensión del papel de los gametos no reducidos en el origen y establecimiento de los neopoliploides en las poblaciones naturales de este complejo.

\section{Conclusiones}

Losresultados delos cruzamientos experimentales entre productores y no productores de gametos no reducidos en T. sidoides subsp. carnea, constituyen el primer aporte para la comprensión del modo de herencia de la capacidad de producir gametos no reducidos en $T$. sidoides. Los mismos sugieren que la capacidad de producir gametos masculinos no reducidos estaría regulada por un gen con dos alelos, uno de ellos completamente dominante sobre el otro, estando el carácter determinado por un alelo recesivo. Sin embargo, diferencias en la expresión génica determinarían la variación en la frecuencia de producción de gametos masculinos no reducidos detectada en la progenie. El hecho de que en $T$. sidoides la capacidad de producir gametos masculinos no reducidos es heredable, contribuiría a aumentar las tasas de formación y de establecimiento de neopoliploides en las poblaciones diploides del complejo.

\section{Agradecimientos}

Las autores agradecen al Ing. Agr. Aveliano Fernández por la lectura crítica del manuscrito. Este trabajo fue financiado por proyectos de la Agencia Nacional de Promoción Científica, Tecnológica y de Innovación (ANPCyT- FONCyT, PICT 07-1329 y 12-1812), y de la Secretaría General de Ciencia y Técnica (UNNE, PI-A003/10). I.E. Kovalsky es becaria Postdoctoral de UNNE-CONICET, y V.G. Solís Neffa es miembro de la Carrera del Investigador Científico de CONICET.

\section{Bibliografía}

ARBO, M.M. 1985. Notas taxonómicas sobre Turneráceas americanas. Candollea 40: 175-191.

AGASHE, B., C. K. PRASAD \& I. SIDDIQI 2002. Identification and analysis of DYAD: a gene 


\section{E. Kovalsky y V. G. Solís Neffa - Análisis de progenie de Turnera sidoides}

required for meiotic chromosome organization and female meiotic progression in Arabidopsis. Development 129: 3935-3943.

BOATENG, K. A., X. YANG, F. DONG, H. A.OWEN \& C. A. MAKAROFF. 2008. SWI1 is required for meiotic chromosome remodeling events. Pl. Molec. Biol. 1: 620-633.

BRETAGNOLLE, F. \& J. D. THOMPSON. 1995. Gametes with the somatic chromosome number: mechanisms of their formation and role in the evolution of autopolyploid plants. New Phytol. 129: $1-22$.

BROWNFIELD, L. \& C. KÖHLER. 2011. Unreduced gamete formation in plants: mechanisms and prospects. J. Exp. Bot. 62: 1659-1668.

CAMADRO, E. L. 1986. Los gametos $2 \mathrm{n}$ en el origen y la evolución de las angiospermas poliploides. Mendeliana 7: 85 - 100.

CAMADRO, E. L. 1994. Second meiotic division restitution (SDR) $2 n$ pollen formation in diploid and hexaploid species of Asparagus.Genet.Resour. Crop Ev. 41: 1-7

D'ERFURTH, I., S. JOLIVET, N. FROGER, O. CATRICE \& M. NOVATCHKOVA. 2008. Mutations in AtPS1 (Arabidopsis thaliana Parallel Spindle 1) lead to the production of diploid pollen grains. PLoS Genet. 4(11): e1000274. doi:10.1371/ journal.pgen.1000274.

D'ERFURTH, I., S. JOLIVET, N. FROGER, O. CATRICE, M. NOVATCHKOVA \& R. MERCIER. 2009. Turning meiosis into mitosis. PLoS Biol. 7: e1000124.

D' ERFURTH, I., L.CROMER, S. JOLIVET, C. GIRARD, C. HORLOW, Y. SUN \& R. MERCIER. 2010. The cyclin-A CYCA1;2/TAM is required for the meiosis I to meiosis II transition and cooperates with OSD1 for the prophase to first meiotic division transition. PLoS Genet. 6: e1000989.

DE STORME, N. \& D. GEELEN. 2011. The Arabidopsis mutant jason produces unreduced FDR male gametes through a parallel/fused spindle mechanism in meiosis II. Pl. Physiol. 155: 1403-1415.

DE HAAN, A., N. O. MACEIRA, R. LUMARET, J. DELAY. 1992. Production of $2 n$ gametes in diploid subspecies of Dactylisglomerata L. 2. Ocurrence and frequency of $2 n$ eggs. Ann. Bot. 69: 345-350.

DE WET, J. M. J. 1980. Origins of polyploids. In: Lewis, W.H. (ed.). Polyploidy, Biological Relevance. Plenum Press, New York. 3-16.

DEWITTE, A., T. EECKHAUT, J. VAN HUYLENBROECK \& E. VAN BOCKSTAELE. 2009. Occurrence of viable unreduced pollen in a Begonia collection. Euphytica 168: 81-94.

DI RIENZO, J.A., F. CASANOVES, M.G. BALZARINI, L. GONZÁLEZ, M. TABLADA \& C.W. ROBLEDO.
2014. InfoStat version 2014. Grupo InfoStat, FCA, Universidad Nacional de Córdoba, Argentina.http:// www.infostat.com.ar.

ELÍAS, G. 2010. Dinámica de una zona de contacto diploide-tetraploide de Turnerasidoidessubsp. pinnatifida (Turneraceae). Tesis Doctoral. Universidad Nacional de Tucumán.

ELÍAS, G., M. SARTOR \& V. G. SOLÍS NEFFA .2011. Patterns of cytotype variation of Turnerasidoides subsp. pinnatifida (Turneraceae) in mountain ranges of central Argentina. J. Plant Res. 124: 25-34.

ERILOVA, A., L. BROWNFIELD, V. EXNER, M. ROSA \& D. TWELL. 2009. Imprinting of the polycomb group gene MEDEA serves as a ploidy sensor in Arabidopsis. PLoS Genet .5(9): e1000663. doi:10.1371/journal.pgen.1000663.

FELBER, F.1991. Establishment of a tetraploidcytotype in a diploid population: effect of relative fitness of the cytotypes. J. Evol. Biol. 4: 195-207.

FERNÁNDEZ, A. 1987. Estudios cromosómicos en Turnera y Piriqueta (Turneraceae). Bonplandia 6:121.

FERNÁNDEZ, A. \& M. M. ARBO. 1990. Gametas no reducidas y relaciones genómicas entre tres especies de Turnera (Turneraceae). Darwiniana 30: 21-26.

FERNÁNDEZ, A. \& M. M. ARBO. 2000a. Cytogenetic relationships between Turneraaurelii, T. cuneiformis $(2 \mathrm{n}=8 \mathrm{x}=40)$ and $T$. orientalis $(2 \mathrm{n}=6 \mathrm{x}=30)$ (Turneraceae). Cytologia 65: 97-102.

FERNÁNDEZ, A. \& M. M. ARBO. 2000b. Relaciones genómicas entre dos especies hexploides de Turnera, T. orientalis y T. velutina, y una diploide, T. grandiflora (Turneraceae, serie Turnera). Bonplandia 10: 181-187.

FERNÁNDEZ, A., H. REY, \& V. G. SOLÍS NEFFA. 2010. Evolutionary relationships between Turnera grandiflora and the octoploid T. fernandezii (Turneraceae). Ann. Bot. Fen. 47: 321-329.

FOWLER N. L. \& D. A. LEVIN. 1984. Ecological constraints on the establishment of a novel polyploid in competition with its diploid progenitor. Amer. Naturalist 124: 703-711.

HARLAN, J. R. \& J. M. J DE WET. 1975. Ö. Winge and a prayer: the origins of polyploidy. Bot. Rev. 41: 361390.

HERMSEM, J. G. T. 1984. Mechanisms and genetic implications of 2 n gamete formation. Iowa State $J$. Res. 58: 421-434.

HUSBAND B. C. \& D. W. SCHEMSKE. 2000. Ecological mechanisms of reproductive isolation between diploid and tetraploid Chamerion angustifolium. J. Ecol. 88: 689-701.

HUSBAND B. C. \& H. A. SABARA .2003.Reproductive isolation between autotetraploids and their diploid progenitors in fireweed, Chamerion angustifolium (Onagraceae).New Phytol. 161: 703-713. 
JONGEDIJK, E. \& M. S. RAMANNA.1988. Synaptic mutants in potato, Solanum tuberosum L. I. Expression and identity of genes for desynapsis. Genome 30: 664-670.

JONGEDIJK, E. \& M. S. RAMANNA. 1989. Synaptic mutants in potato, Solanum tuberosumL. II. Concurrent reduction of chiasma frequencies in male and female meiosis of ds-1 (desynapsis) mutants. Genome 32: 1054-1062.

JONGEDIJK, E., J. M. A. S. A. VANDERWOLK \& L. C. J. M. SUURS. 1990. Analysis of glutamate oxaloacetate transaminase (GOT) isozyme variants in diploid tuberous Solanum; inheritance and linkage relationships to $d s$ (desynapsis), $y$ (tuber flesh colour), $c r$ (crumpled) and $y c$ (yellow cotyledon). Euphytica 45: 155-167.

KATSIOTIS, A. \& R. A. FORSBERG. 1995. Discovery of $2 \mathrm{n}$ gametes in tetraploid Avenavaviloviana. Euphytica 81: 1-6.

KAUL, M. L. H. \& T. G. K. MURTHY. 1985. Mutant genes affecting higher plant meiosis. Theor. Appl. Genet. 70: 449-466.

KOVALSKY, I. E. 2012. Origen y establecimiento de neopoliploides en poblaciones naturales de Turnera sidoides L. (Turneraceae).Tesis Doctoral, Universidad Nacional de Córdoba.

KOVALSKY, I. E. \& V. G. SOLÍS NEFFA. 2012. Evidence of $2 \mathrm{n}$ microspore production in a natural diploid population of Turnera sidoides subsp. carnea and its relevance in the evolution of the $T$. sidoides (Turneraceae) autopolyploid complex. J. Pl. Res. 125: 725-734.

KOVALSKY, I. E., A. FERNANDEZ \& V. G. SOLÍS NEFFA. 2014. Mecanismos citológicos involucrados em la producción de gametos masculinos no reducidos en indivíduos diploides de Turnera sidoides subsp. carnea (Passifloraceae).Bol. Soc. Argent. Bot. 49: 227-234.

LAZAROFF, Y. A., A. FERNÁNDEZ \& V. G. SOLÍS NEFFA. 2010. Análisis de la producción y frecuencia de gametos no reducidos en poblaciones diploides de Turnera krapovickassi (Turneraceae). J. BAG XLI (Suplemento): 118.

LEVIN, D. A. 1975. Minority cytotype exclusion in local plant populations. Taxon.24: 35-43.

LEVIN, D. A .1983. Polyploidy and novelty in flowering plants. Amer Naturalist. 122: 1-25.

MAIZONNIER, D. 1976. Production de tretraploides et de trisomiques naturelz chez le Petunia. Annales d'Amélioration des Plantes 26: 305-318.

MC COY, T.J. 1982. Inheritance of $2 \mathrm{n}$ pollen formation in diploid alfalfa (Medicago sativa L.). Canad. J. Genet. Cytol. 24: 315-323.

MENDIBURU, A. O. \& S. J. PELOQUIN. 1977. The significance of $2 n$ gametes in potato breeding. Theor. Appl. Genet. 49: 53-61.
MERCIER, R., D. VEZON, E. BULLIER, J. C MOTAMAYOR., A. SELLIER, F. LEFÈVRE, \& C. HORLOW. 2001. SWITCH1 (SWI1): a novel protein required for the establishment of sister chromatid cohesion and for bivalent formation at meiosis. Annales d'Amélioration des Plantes 15: 1859-1871.

MERCIER, R., S. J. ARMSTRONG, C. HORLOW, N. P. JACKSON, C. A. MAKAROFF, D. VEZON \& F. C. H. FRANKLIN. 2003. The meiotic protein SWI1 is required for axial element formation and recombination initiation in Arabidopsis. Development 130: 3309-3318.

MOK, D. W. S. \& S. J. PELOQUÍN. 1975. Three mechanisms of $2 \mathrm{n}$ pollen formation in diploid potatoes. Can. J. Genet.Cytol. 17: 217-225.

ORJEDA, G., R. FREYRE \& M. IWANAGA .1990. Production of $2 n$ pollen in diploid Ipomoea trifida, a putative wild ancestor of the sweet potato.J. Hered. 81: 462-467.

ORTIZ, R. 1996. Ocurrence and inheritance of $2 \mathrm{n}$ pollen in Musa. Ann. Bot. 79: 449- 453.

ORTIZ, R. \& S.J. PELOQUÍN. 1992. A new method of producing inexpensive $4 \mathrm{x}$ hybrid true potato seed. Euphytica 57: 103-108.

PAN, G., Y. ZHOU, L. C. FOWKE \& H. WANG. 2004. An efficient method for flow cytometric analysis of pollen and detection of $2 \mathrm{n}$ nuclei in Brassica napus pollen. Pl. Cell Rep. 23: 196-202.

PANSERI, A. F. 2011. Estructura genética y variación de los caracteres reproductivos en Turnera sidoides subsp. pinnatifida (Turneraceae). Tesis Doctoral. Universidad Nacional del Nordeste.

PANSERI, A. F., J. G. SEIJO \& V. G. SOLÍS NEFFA. 2008. Análisis de la producción y frecuencia de microsporas no reducidas en diploides de Turnerasidoides (Turneraceae). Bol. Soc. Argent. Bot. 43: 95-101.

PARROT, W. A. \& R. R. SMITH. 1985. Production of $2 \mathrm{n}$ pollen in red clover. Crop Sci. 24: 469-472.

QU, L. \& N. VORSA. 1999. Desynapsis and spindle abnormalities leading to $2 n$ pollen formation in Vaccinium darrowii. Genome 42: 35-40.

RAMANNA, M. S. 1983. First division restitution gametes through fertile desynaptic mutants of potato. Euphytica 32: 337-350.

RAMSEY, J. \& D. W. SCHEMSKE. 1998. Pathways, mechanisms, and rates of polyploid formation in flowering plants. Annu. Rev. Ecol. System. 29: 467501.

SCHATLOWSKI, N. \& C. KÖHLER. 2012. Tearing down barriers: understanding the molecular mechanisms of interploidy hybridizations. J. Exp. Bot. 63: 60596067.

SOLÍS NEFFA, V. G. 2000. Estudios biosistemáticos en el complejo Turnera sidoides L. (Turneraceae, 


\section{E. Kovalsky y V. G. Solís Neffa - Análisis de progenie de Turnera sidoides}

Leiocarpae). Tesis Doctoral, Universidad Nacional de Córdoba.

SOLÍS NEFFA, V. G. \& A. FERNÁNDEZ. 2000. Chromosome studies in Turnera (Turneraceae).2000. Genetics and Molecular Biology 23: 925-930.

SOLÍS NEFFA, V. G. \& A. FERNÁNDEZ. 2001. Cytogeography of the Turnera sidoides L. complex (Turneraceae, Leiocarpae). Bot. J. Linn. Soc. 137: 189-196.

SOLÍS NEFFA, V. G., A. F. PANSERI, W. REYNOSO \& J. G. SEIJO.2004. Variación en el color de flores y números cromosómicos en el Noroeste del área de distribución de Turnera sidoides (Turneraceae). Bonplandia 13: 117-128.

TAVOLETTI, S., A. MARIANI \& F. VERONESI. 1991. Phenotypic recurrent selection for $2 n$ pollen and $2 n$ egg production in diploid alfalfa. Euphytica 57 : 97-102.

TAVOLETTI, S., P. PESARESI, G. BARCACCIA, E. ALBERTINI \& F. VERONESI. 2000. Mapping the jp (jumbo pollen) gene and QTLs involved in multinucleate microspore formation in diploid alfalfa. Theor. Appl. Genet .101: 372-378.

THOMPSON J. D. \& R. LUMARET .1992. The evolutionary dynamics of polyploid plants: origins, establishment and persistence. Trends Ecol. Evol. 7: 302-307

VAN CAMPO, M. 1957. Palynologie africaine I. Bulletin de l'Institut Français d'Afrique Noire 19: 659-678.

VAN DIJK, P. \& R. BIJLSMA. 1994. Simulations of flowering time displacement between cytotypes that form inviable hybrids. Heredity 72: 522-535.
DIJK, P. V., M. HARTOG \& W. V. DELDEN. 1992. Single cytotype areas in autopolyploid Plantago media L. Biol. J. Linn. Soc..46: 315-331.

VEERLE, L., J. BAERT, I. ROLDAN-RUIZ, M. DE LOOSE \& E. VAN BOCKSTAELE. 2002. Tracing of $2 n$ egg occurrence in perennial rye grass (Loliumperenne L.) using interploidy crosses. Euphytica 123: 159-164.

VEILLEUX, R.E. 1985. Diploid and polyploid gametes in crop plants: mechanisms of formation and utilization in plant breeding. Plant Breed. Rev. 3: 253-288.

VEILLEUX, R. E. \& F. I. LAUER. 1981. Variation for 2n pollen production in clones of Solanum phureja Juz. and Buk. Theor. Appl. Genet. 59: 95-100.

VEILLEUX, R. E., N. A. MC HALE \& F. I. LAUER .1982.Unreduced gametes in diploid Solanum. Frequency and types of spindle abnormalities. Canad. J. Genet. Cytol 24: 301-314.

VERONESI, F., S. TAVOLETTI \& A. MARIANI. 1990. Identification of $2 \mathrm{n}$ and $4 \mathrm{n}$ gamete producers in an experimental population of diploid Medicago. $J$. Genet. Breed. 44: 143-148.

WATANABE K. \& S. J. PELOQUÍN.1989. Ocurrence of $2 \mathrm{n}$ pollen and ps gene frequencies in cultivated groups and their related wild species in tuber-bearing Solanums. Theor. Appl. Genet. 78: 329-336.

Recibido el 30 de septiembre de 2014, aceptado el 17 de noviembre de 2014. 
